\title{
A Case of Locally Advanced Breast Cancer Successfully Treated with Multidisciplinary Therapy
}

\author{
Masafumi Tomita $^{a}$ Shoji Ourab Shinichiro Makimoto ${ }^{a}$ \\ aDepartment of Surgery, Kishiwada Tokushukai Hospital, Kishiwada, Japan; \\ ${ }^{b}$ Division of Breast Surgery, Kishiwada Tokushukai Hospital, Kishiwada, Japan
}

\section{Keywords}

Multidisciplinary treatment · Locally advanced breast cancer · Luminal breast cancer

\begin{abstract}
A 42-year-old woman with locally advanced luminal breast cancer was referred to our hospital. Ultrasonography showed marked skin thickening with obscured breast mass. Positron emission tomography showed bilateral swelling in the axillar, parasternal, and supraclavicular lymph nodes in addition to the primary breast cancer. Following the patient's request, we treated her with primary bevacizumab-containing chemotherapy followed by radiotherapy to the breast due to the histologically proven widely spreading cancer remnants in the skin after chemotherapy. Thereafter, the patient underwent mastectomy and axillary dissection with a vertical rectus abdominis musculo-cutaneous flap to cover the skin defect. Pathological examination showed no viable cancer cells both in the breast and lymph nodes but imperceptible cytokeratin AE1 + 3-positive cells in the skin. Although wound healing needed 3 months due to the influence of preoperative radiotherapy, the patient has been well on endocrine therapy without any recurrence for more than 7 years.

\section{Introduction}

Due to the prevalence of mammographic screening, the mortality rate of breast cancer has dropped in developed Western countries $[1,2]$. On the other hand, despite the awareness of breast mass, some patients with breast cancer do not consult a doctor for a long time. Various disgusting symptoms such as pungent smell and bleeding make them unwillingly visit doctors, resulting in their breast masses to be diagnosed as locally advanced breast cancers with or without distant metastasis. Chemotherapy alone or with anti-human 
Tomita et al.: Advanced Breast Cancer Successfully Treated with Multidisciplinary Therapy

Fig. 1. Local findings. a Before treatment, multiple satellite skin lesions and skin edema were observed. $\mathbf{b}$ After chemotherapy, satellite skin lesions regressed markedly, but skin edema and pigmentation were still observed. c After radiotherapy, slight skin pigmentation was still observed, but almost all of the satellite skin lesions had disappeared. d Ten months after operation, a vertical rectus abdominis muscle flap covered the large skin defect and offered favorable cosmetic outcome.

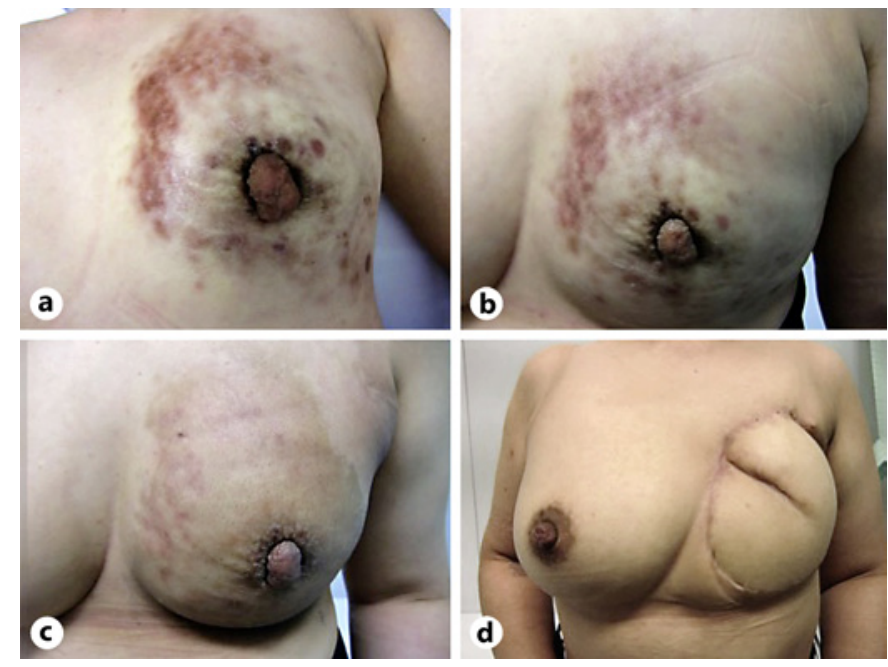

epidermal growth factor receptor type 2 (HER2) agent is a feasible therapeutic approach for patients with triple negative and HER2 type locally advanced breast cancer, respectively. Endocrine therapy is often applied in patients with luminal type locally advanced breast cancer to preserve quality of life of these patients. We report here a case of luminal type locally advanced breast cancer successfully treated with multidisciplinary therapy consisting of chemotherapy, radiotherapy, surgery, and endocrine therapy.

\section{Case Report}

A 42-year-old woman with huge mass and skin thickening in her left breast (Fig. 1a) was referred to our hospital. Mammography was not done due to the hardness of the affected left breast. Ultrasonography showed marked skin thickening with obscured breast mass (Fig. 2a) and enlarged axillary lymph nodes (Fig. 2c). Core needle biopsy showed the tumor to be a luminal (estrogen receptor positive; Allred score 8, progesterone receptor negative) invasive ductal carcinoma. In addition to her left breast cancer, positron emission tomography showed bilateral swelling in the axillar, parasternal, and supraclavicular lymph nodes without visceral metastases. Contralateral axillar lymph node metastasis was pathologically confirmed by fine-needle aspiration cytology. Taking the Allred score and disease spread into consideration, we offered the patient primary endocrine therapy with palliative intent. The patient, however, requested to undergo multidisciplinary therapy with curative intent.

After obtaining informed consent that primary chemotherapy might cause some decrease in endocrine sensitivity of the tumor, we treated the patient with primary bevacizumabcontaining chemotherapy; weekly paclitaxel $\left(80 \mathrm{mg} / \mathrm{m}^{2} \mathrm{qw} \times 12\right)$ and bi-weekly bevacizumab $(10 \mathrm{mg} / \mathrm{kg}$ ) therapy followed by FEC100 (fluorouracil $500 \mathrm{mg}$, epirubicin $100 \mathrm{mg}$, and cyclophosphamide $500 \mathrm{mg} / \mathrm{m}^{2} \mathrm{q} 3 \mathrm{w} \times 4$ ) and tri-weekly bevacizumab (15 mg/kg) therapy. Primary chemotherapy resulted in a marked regression of the breast tumor (Fig. 1b) and complete remission of the regional nodes. However, punch biopsy of the still swollen skin after primary chemotherapy showed widely spreading viable cancer remnants. Therefore, radiotherapy (48 Gy, 24 fractions) was applied to the whole breast to eradicate cancer cells in the skin, leading to further improvement of the skin edema (Fig. 1c). The patient underwent mastectomy with possible removal of the skin overlying the mammary gland and ipsilateral axillary lymph node dissection 2 months after completing radiotherapy. 
Fig. 2. Ultrasound images. a Before treatment, marked skin thickening with obscured breast mass was observed. b After chemotherapy, although skin thickening was still observed, a marked shrinkage of the breast tumor (yellow arrow) was observed. c Before treatment, enlarged and oval axillary lymph nodes (white arrow) were observed. d After chemotherapy, markedly regressed lymph nodes (red arrow) were observed.
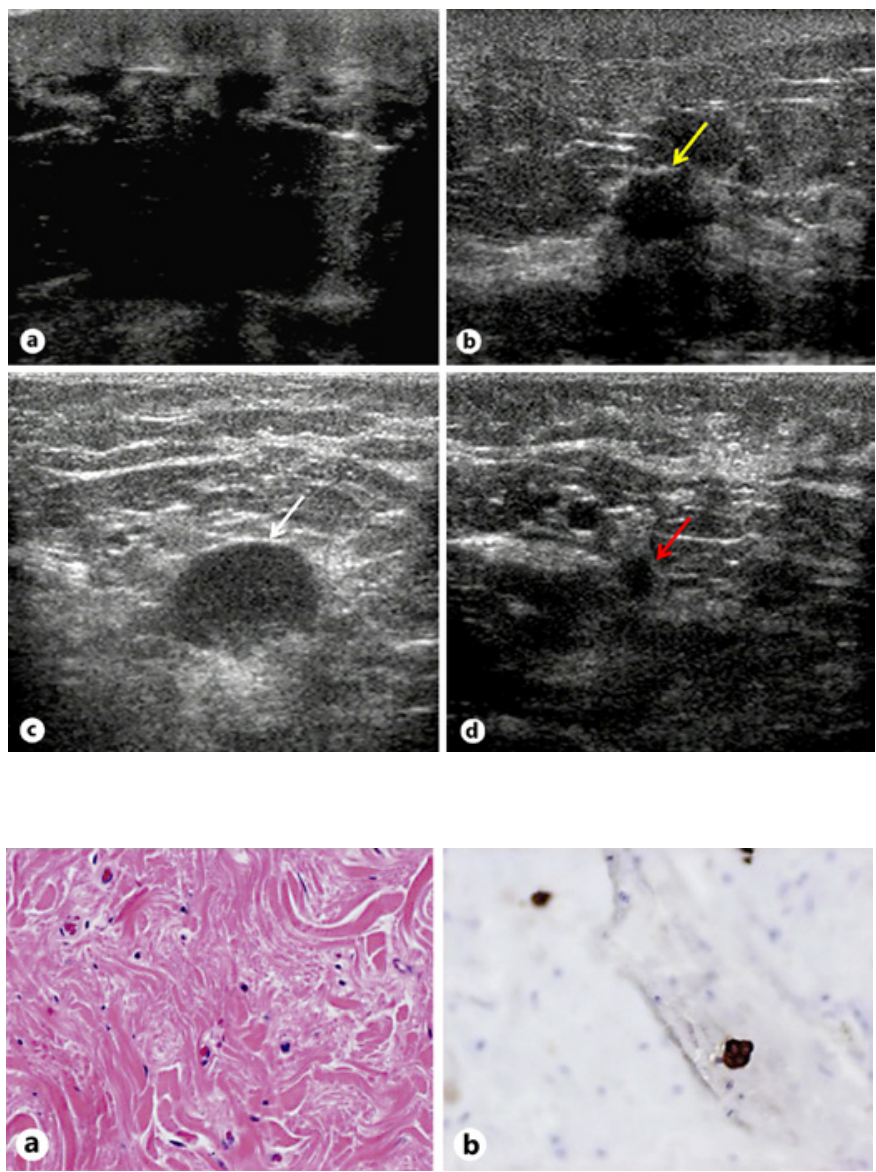

Fig. 3. Pathological findings. a Marked fibrosis and no viable cancer cells were observed on HE staining of the tumor $(\times 40)$. $\mathbf{b}$ Isolated and atypical cells were observed on cytokeratin AE1 +3 staining $(\times 100)$ of the skin near the nipple-areolar complex.

In order to achieve a direct closure of the skin defect, we used a musculo-cutaneous (MC) flap using her vertical rectus abdominis muscle. Pathological examination showed negative surgical margin and no viable cells both in the breast and lymph nodes (Fig. 3a) but cytokeratin AE1 + 3-positive imperceptible cells in the resected skin (Fig. 3b). Due to the influence of preoperative radiotherapy to the breast, wound healing needed 3 months. The patient has received aromatase inhibitor with luteinizing-hormone-releasing hormone agonist and has been well without any recurrence for more than 7 years (Fig. 1d).

\section{Discussion}

Metastatic breast cancer can hardly be cured and is often treated by treatments with palliative intent to alleviate or prevent breast cancer-induced unpleasant symptoms and possibly prolong survival of the patient. Metastatic breast cancer, therefore, is generally managed with the treatments according to the sequence proposed by Hortobagyi [3]: nonlife-threatening and hormone-responsive breast cancer should first be treated with endocrine therapy to maintain quality of life of the patient. However, if treatments based on Hortobagyi's algorithm had been applied to our patient, they could, on the one hand, have avoided chemotherapy-induced unpleasant side effects in this patient initially, but they could never have brought about the presumed cure, on the other hand.

It is well known that luminal breast cancer shows a smaller effect [4] of chemotherapy than triple-negative breast cancer [5] and HER2-positive breast cancer [6]. Therefore, we 
intended in advance to apply some kind of local therapy to the patient after completing chemotherapy at the time to develop a therapeutic strategy. In fact, bevacizumab-containing chemotherapy resulted in clinical complete response in the regional nodes but widely spreading cancer residuals in the skin pathologically, leading to the application of radiotherapy to the breast prior to surgery.

To cover the skin defect, we used an MC flap using her vertical rectus abdominis muscle [7]. Except for a vertical rectus abdominis muscle MC flap, we could have selected a split/full thickness skin graft, latissimus dorsi MC flap [8], or a transverse rectus abdominis muscle MC flap [9]. We, however, did not select a split/full thickness skin graft, latissimus dorsi MC flap, or a transverse rectus abdominis muscle MC flap due to the high possibility of wound healing failure due to pre-operative radiotherapy, the limitation of the applicable skin size, and unstable blood supply, respectively. We think this selection was appropriate due to the fact that even the vertical rectus abdominis MC flap, generally regarded as one of the flaps with stable blood supply, needed 3 months for wound healing.

Routine pathological examination of the resected specimen showed that no viable cancer cells were observed in the breast and the axillar lymph nodes. Immunohistochemical study using cytokeratin AE1 + 3 staining showed imperceptible atypical cells in the skin near the nipple-areolar complex. Neither radiotherapy nor surgery was applied to the bilateral parasternal and supraclavicular nodes, and no recurrence was observed in these areas. We, therefore, cannot judge whether these cytokeratin AE1 + 3-positive cells could have led to skin recurrence or not, if surgical intervention had not been done to the breast.

In conclusion, we cannot suppose what clinical outcome would have been achieved if a therapeutic strategy based on Hortobagyi's algorithm had been applied in this case. Clinical outcome in this case, however, suggests that multidisciplinary therapy with curative intent [10-12] should be taken into consideration during decision making on how to treat inoperable luminal breast cancer.

\section{Statement of Ethics}

We have reported this case in compliance with the Declaration of Helsinki. Informed consent was obtained from the patient for the publication of the clinical data.

\section{Disclosure Statement}

The authors have no conflicts of interest to declare.

\section{Funding Sources}

No funding was received for this research.

\section{Author Contributions}

All authors contributed equally to this work. 


\section{References}

1 Habbema JD, van Oortmarssen GJ, van Putten DJ, Lubbe JT, van der Maas PJ. Age-specific reduction in breast cancer mortality by screening: an analysis of the results of the Health Insurance Plan of Greater New York study. J Natl Cancer Inst. 1986;77:317-20.

2 Nyström L, Andersson I, Bjurstam N, Frisell J, Nordenskjöld B, Rutqvist LE. Long-term effects of mammography screening: updated overview of the Swedish randomised trials. Lancet. 2002 16;359:909-19.

3 Hortobagyi GN. Treatment of breast cancer. N Engl J Med. 1998;339:974-84.

4 Toi M, Nakamura S, Kuroi K, Iwata H, Ohno S, Masuda N, et al. Phase II study of preoperative sequential FEC and docetaxel predicts of pathological response and disease free survival. Breast Cancer Res Treat. 2008;110:531-9.

5 Lehmann BD, Jovanović B, Chen X, Estrada MV, Johnson KN, Shyr Y, et al. Refinement of triple-negative breast cancer molecular subtypes: implications for neoadjuvant chemotherapy selection. PLoS One. 2016;11: e0157368.

6 Buzdar AU, Ibrahim NK, Francis D, Booser DJ, Thomas ES, Theriault RL, et al. Significantly higher pathologic complete remission rate after neoadjuvant therapy with trastuzumab, paclitaxel, and epirubicin chemotherapy: results of a randomized trial in human epidermal growth factor receptor 2 - positive operable breast cancer. J Clin Oncol. 2005;23:3676-85.

7 Houdek MT, Bakri K, Tibbo ME, Wagner ER, Rose PS, Sim FH, et al. Outcome and complications following vertical rectus abdominis myocutaneous flap surgery to reconstruct sacrectomy defects. Plast Reconstr Surg. 2018;142:1327-35.

8 Sood R, Easow JM, Konopka G, Panthaki ZJ. Latissimus dorsi flap in breast reconstruction: recent innovations in the workhorse flap. Cancer Control. 2018;25:1073274817744638.

9 Wilkins EG, August DA, Kuzon WM Jr, Chang AE, Smith DJ. Immediate transverse rectus abdominis musculocutaneous flap reconstruction after mastectomy. J Am Coll Surg. 1995;180:177-83.

10 Milano MT, Zhang H, Metcalfe SK, Muhs AG, Okunieff P. Oligometastatic breast cancer treated with curativeintent stereotactic body radiation therapy. Breast Cancer Res Treat. 2009;115:601-8.

11 Kwapisz D. Oligometastatic breast cancer. Breast Cancer. 2019;26:138-46.

12 Westphal T, Gampenrieder SP, Rinnerthaler G, Greil R. Cure in metastatic breast cancer. Memo. 2018;11:172-9. 